To be submitted to ApJL

\title{
SIMULATIONS OF PROMINENCE FORMATION IN THE MAGNETIZED SOLAR CORONA BY CHROMOSPHERIC HEATING
}

\author{
C. Xia ${ }^{1}$, P. F. Chen ${ }^{1}$, R. Keppens ${ }^{2}$
}

\begin{abstract}
Starting from a realistically sheared magnetic arcade connecting chromospheric, transition region to coronal plasma, we simulate the in-situ formation and sustained growth of a quiescent prominence in the solar corona. Contrary to previous works, our model captures all phases of the prominence formation, including the loss of thermal equilibrium, its successive growth in height and width to macroscopic dimensions, and the gradual bending of the arched loops into dipped loops, as a result of the mass accumulation. Our 2.5-dimensional, fully thermodynamically and magnetohydrodynamically consistent model mimics the magnetic topology of normal-polarity prominences above a photospheric neutral line, and results in a curtain-like prominence above the neutral line through which the ultimately dipped magnetic field lines protrude at a finite angle. The formation results from concentrated heating in the chromosphere, followed by plasma evaporation and later rapid condensation in the corona due to thermal instability, as verified by linear instability criteria. Concentrated heating in the lower atmosphere evaporates plasma from below to accumulate at the top of coronal loops and supply mass to the later prominence constantly. This is the first evaporation-condensation model study where we can demonstrate how the formed prominence stays in a force balanced state, which can be compared to the Kippenhahn-Schlüter type magnetohydrostatic model, all in a finite low-beta corona.
\end{abstract}

Subject headings: Sun: filaments, prominences — Sun: corona

\footnotetext{
${ }^{1}$ School of Astronomy and Space Science, Nanjing University, Nanjing 210093, China; chenpf@nju.edu.cn

${ }^{2}$ Centre for Plasma Astrophysics, Department of Mathematics, K.U.Leuven, Celestijnenlaan 200B, 3001 Heverlee, Belgium
} 


\section{INTRODUCTION}

Prominences represent fascinating large-scale, cool $(\sim 8000 \mathrm{~K})$ and dense $\left(10^{10} \sim 10^{11}\right.$ $\mathrm{cm}^{-3}$ ) structures, suspended in the hot and tenuous solar corona above magnetic neutral lines, which separate opposite polarity photospheric magnetic regions. The magnetic field strength in quiescent prominences lies between 3-15 G (Leroy et al. 1983). It is much stronger in active region prominences. The field topology of prominences is less accurately known, but was found to be mostly horizontal, making an acute angle with respect to the axis of the prominence (Bommier \& Leroy 1998). It is accepted that the stable existence of prominences is due to the mechanical support and thermal shielding of the magnetic fields. Two topologically different types of static prominence models were proposed by Kippenhahn \& Schlüter (1957) and Kuperus \& Raadu (1974). In the Kippenhahn-Schlüter (K-S) prominences, also known as normal-polarity prominences, the horizontal magnetic field through the prominence points from the underlying positive to the negative magnetic polarities in the photosphere. In Kuperus-Raadu $(\mathrm{K}-\mathrm{R})$ prominences, or inverse-polarity prominences, the magnetic field points from negative to positive. In both types, the concaveupward parts of magnetic field lines or 'dips' host and support prominence material via the magnetic tension force against gravity. These have been extended by many authors (Low) 1975; Amari \& Aly 1989; Hood \& Anzer 1990), and recently, flux-rope embedded, normaland inverse- polarity type equilibrium configurations have been amenable to numerical modeling (Petrie et al. 2007; Blokland \& Keppens 2011). These models merely consider magnetohydrostatic force balance arguments, and leave out the thermodynamic aspects.

The most elusive aspect in prominence physics is their seemingly in-situ formation. One of the earliest suggested formation scenarios relates the sudden appearance of prominence material to a radiative condensation process due to thermal instability (Parker 1953; Field 1965). In the optically thin solar corona, the radiative losses are proportional to density squared and the temperature can evolve into the critical range where a decrease of temperature leads to an increase of the radiative losses (Colgan et al. 2008). When the plasma is perturbed to become dense or cool enough for radiative losses to dominate both the heating by thermal conduction and any background heating process, sudden strong cooling can cause sufficient pressure gradient to suck in material around along field lines. Hence, plasma condenses into cool regions and settles in magnetic dips to form largescale prominences. The linear 'trigger' of such thermal-instability condensations was studied in multi-dimensional sheared magnetic field by many authors (van Hoven \& Mok 1984; van der Linden et al. 1992). The nonlinear evolution of such thermal instability can only be researched by numerical simulations. Hildner (1974) firstly performed two-dimensional (2D) numerical simulations of the thermal instability for the formation of prominences in a uniform magnetic and gravitational field, while neglecting thermal conduction. Sparks et al. 
(1990) simulated a nonlinear condensation in a force-free sheared magnetic field including anisotropic thermal conduction, but neglected gravity. Drake et al. (1993) simulated the formation and stable support of a prominence at the apex of a potential magnetic arcade including the upper chromosphere. But in their simulations, the initial trigger perturbations were artificially added without any detailed mechanism ultimately giving rise to condensation into a prominence.

Many mechanisms for the transport of plasma from the chromosphere to the corona thereby triggering the formation of prominences have meanwhile been proposed(see the review by Mackay et al. 2010). An et al. (1988) and Wu et al. (1990) simulated the formation of a prominence in a potential field arcade in an initially isothermal corona by injecting high density material ballistically from the bottom boundary. Choe \& Lee (1992) performed a 2.5-dimensional (2.5D) magnetohydrodynamic (MHD) simulation to investigate prominence formation due to siphon flows by photospheric shearing motions. These simulations did not include photosphere or chromosphere regions. One-dimensional (1D) evaporationcondensation model, which include chromosphere and chromospheric heating, have been studied numerically by many authors (Mok et al. 1990; Antiochos et al. 1999; Karpen et al. 2001; Xia et al. 2011). This thermo-hydrodynamic model depends on heating localized in the chromospheric feet of a pre-shaped loop, which evaporates chromospheric material into the corona. The increase in density results in a dominating radiative cooling, and the thermal instability triggers condensations in the corona, eventually forming a steadily growing or recycling prominence (Xia et al. 2011). In this Letter, we present the first simulation where this model is performed in a 2.5D full MHD regime in a realistically stratified sheared arcade going from the low chromosphere to high corona. We show how we can simulate the rapid thermodynamical changes in a scenario where we form and reach a stable support for a $\mathrm{K}-\mathrm{S}$ quiescent prominence.

\section{NUMERICAL SETUP}

We perform our simulation in a rectangular, Cartesian geometry, ignoring the curvature of the solar surface. Since prominences are often observed in a horizontally elongated form along the magnetic neutral line, we ignore the variation of physical variables along the prominence axis ( $z$-direction) and consider their dependence on horizontal $x$-coordinate (perpendicular to the magnetic neutral line) and vertical $y$-coordinate, while keeping the $z$-component of any vector. Therefore, we use a 2.5D thermodynamic MHD model including gravity, field-aligned heat conduction, radiative cooling and heating terms. The governing 
equations are as follows

$$
\begin{gathered}
\frac{\partial \rho}{\partial t}+\nabla \cdot(\rho \mathbf{v})=0 \\
\frac{\partial(\rho \mathbf{v})}{\partial t}+\nabla \cdot\left(\rho \mathbf{v} \mathbf{v}+p_{t o t} \mathbf{I}-\mathbf{B B}\right)=\rho \mathbf{g} \\
\frac{\partial E}{\partial t}+\nabla \cdot\left(E \mathbf{v}+p_{t o t} \mathbf{v}-\mathbf{B B} \cdot \mathbf{v}\right)=\rho \mathbf{g} \cdot \mathbf{v}+\nabla \cdot(\boldsymbol{\kappa} \cdot \nabla T)-Q+H \\
\frac{\partial \mathbf{B}}{\partial t}+\nabla \cdot(\mathbf{v B}-\mathbf{B v})=0
\end{gathered}
$$

where $\rho, T, \mathbf{v}, \mathbf{B}$, and $\mathbf{I}$ are the plasma density, temperature, velocity, magnetic field, and unit tensor, respectively; $p_{t o t} \equiv p+B^{2} / 2$ is the total pressure, composed of thermal pressure $p$ and magnetic pressure $B^{2} / 2 ; E=p /(\gamma-1)+\rho v^{2} / 2+B^{2} / 2$ is the total energy density, where $\gamma=5 / 3$ is the ratio of specific heats; $\mathbf{g}=-g_{0} R_{\odot}^{2} /\left(R_{\odot}+y\right)^{2} \hat{\mathbf{y}}$ is the gravitational acceleration with $R_{\odot}$ the solar radius and $g_{0}$ the solar surface gravitational acceleration; $\boldsymbol{\kappa}$ is the thermal conductivity tensor; $Q$ and $H$ are the radiative loss rate and heating densities, respectively. Considering fully ionized plasma with 10:1 abundance of hydrogen and helium, we have $\rho=1.4 m_{p} n_{\mathrm{H}}$, where $m_{p}$ is the proton mass and $n_{\mathrm{H}}$ is the number density of hydrogen. As for the equation of state, we adopt the ideal gas law $p=2.3 n_{\mathrm{H}} k_{B} T$, where $k_{B}$ is the Boltzmann constant. The radiative cooling term is taken as $Q=1.2 n_{\mathrm{H}}^{2} \Lambda(T)$, where $\Lambda(T)$ is the radiative loss function for optically thin emission, which was also used in our earlier 1D studies (Xia et al. 2011). The anisotropic thermal conduction along the magnetic field lines is included with $\boldsymbol{\kappa}=\kappa \hat{\mathbf{b}} \hat{\mathbf{b}}$, where $\hat{\mathbf{b}}$ is the unit vector along $\mathbf{B}$ and $\kappa=10^{-6} T^{5 / 2} \mathrm{erg} \mathrm{cm}^{-1}$ $\mathrm{s}^{-1} \mathrm{~K}^{-1}$ is the Spitzer conductivity.

As for the initial magnetic field, we intend to mimic sheared arcades above a neutral line $(x=0, y=0)$ and start our simulation from a force-balanced state. Therefore we adopt an analytic solution of a nonlinear force-free field found by Low (1977) as follows

$$
\begin{gathered}
B_{x}=2 B_{0}\left(k y+\frac{1-\mu^{2}}{1+\mu^{2}}\right) / f, \quad B_{y}=-2 B_{0} k x / f, \quad B_{z}=\frac{4 \mu B_{0}}{1+\mu^{2}} / f, \\
\text { where } f=\frac{4 \mu^{2}}{\left(1+\mu^{2}\right)^{2}}+k^{2} x^{2}+\left(k y+\frac{1-\mu^{2}}{1+\mu^{2}}\right)^{2},
\end{gathered}
$$

where $|\mu|<1$ controls the shearing rate of the arcades (no shearing if $\mu=0$ ) and $k$ controls the spatial concentration of the field. The shearing decays from lower loops to higher loops. We set $\mu=0.95, k=0.5$, and $B_{0}=4 \mathrm{G}$, leading to a realistic $2.5 \mathrm{D}$ arcade topology, where the field lines at the height of $20 \mathrm{Mm}$ make an angle of $45^{\circ}$ with the underlying neutral line.

For the initial thermal structure, we set a chromosphere with temperature of 10000 $\mathrm{K}$ below a height of $2.7 \mathrm{Mm}$ and choose a vertically stratified temperature profile with a 
constant vertical thermal conduction flux (i.e. $\kappa \partial T / \partial y=2 \times 10^{5} \mathrm{ergs}^{-2} \mathrm{~s}^{-1}$ ) above the height (Mok et al. 2005; Fontenla et al. 1991). The initial density is then determined by assuming a hydrostatic atmosphere with the number density of $2 \times 10^{13} \mathrm{~cm}^{-3}$ at the bottom. To use a proper background heating term to maintain a hot corona, we are inspired by parametric comparisons of different models of coronal heating done by Mandrini et al. (2000) and assume the heating rate to be proportional to $B^{2}$ (Mok et al. 2008). However, in order to compensate the radiative loss in the transition region above the neutral line, where dominating horizontal field lines insulate this region from getting heat thermally conducted from the corona above, we add an extra heating equal to the local radiative loss, below a $6 \mathrm{Mm}$ height, purely concentrated in this region. The resulting two-component, parametric background heating is expressed as

$$
H_{0}=c_{0} B^{2}+0.5 Q \cos \left(\frac{\pi B_{y}}{2 B_{0}}\right)\left[1-\tanh \left(\frac{y-y_{h}}{y_{d}}\right)\right],
$$

with $c_{0}=7.5 \times 10^{-6} \mathrm{erg} \mathrm{cm}^{-3} \mathrm{~s}^{-1} \mathrm{G}^{-2}, y_{h}=6 \mathrm{Mm}$, and $y_{d}=0.5 \mathrm{Mm}$.

The configuration of this system is symmetric about the $y$-axis. We exploit the symmetry to study prominence formation under symmetric heating conditions, allowing to reduce computational domain to the right half of the simulated area (within $0<x<30 \mathrm{Mm}$ and $0<y<40 \mathrm{Mm}$ ). Symmetric/asymmetric boundary treatments can then be used at the $y$-axis, while for right-hand side boundaries we adopt a zero velocity, continuous density and pressure, and fixed magnetic field. The top and the bottom boundaries have a zero velocity, fixed magnetic field and extrapolated density and pressure, respectively, which are derived by assuming a hydrostatic equilibrium with the temperature being fixed.

We use the parallelized Adaptive Mesh Refinement Versatile Advection Code (MPIAMRVAC) (Keppens et al. 2012) to solve the governing equations with a second-order shockcapturing Total Variation Diminishing Lax-Friedrichs scheme. The effective resolution of $512 \times 1024$ is attained by using 5 levels of AMR. The equivalent spatial resolution is then $39 \mathrm{~km} / 59 \mathrm{~km}$ in the vertical/horizontal direction. Anisotropic thermal conduction is added explicitly as an energy source term.

This initial state is not in thermal equilibrium, and we integrate the governing equations in time with the background heating $H=H_{0}$ until the system relaxes to a quasi-equilibrium shown by panels (a) and (b) in Figure 1. The chromosphere of about $4 \mathrm{Mm}$ thickness is connected to the corona by a very thin transition region. The projected magnetic field lines, colored according to the local density, are plotted through selected footpoints at the bottom and side boundaries. The plasma beta is 0.1 at $20 \mathrm{Mm}$ height above the neutral line while the temperature and number density are $1.6 \mathrm{MK}$ and $2.6 \times 10^{8} \mathrm{~cm}^{-3}$ there, respectively. The maximal residual velocity is small, less than $5 \mathrm{~km} \mathrm{~s}^{-1}$. Starting from this quasi-equilibrium, a 
relatively strong heating $H_{1}$ is added. This extra heating is localized near the chromosphere (see the contours in Figure 1(a)) with its formula as:

$$
H_{1}= \begin{cases}C_{1}\left(B_{y} / B_{0}\right)^{2} & \text { if } y \leqslant y_{c} \\ C_{1}\left(B_{y} / B_{0}\right)^{2} \exp \left(-\left(y-y_{c}\right)^{2} / \lambda\right) & \text { if } y>y_{c}\end{cases}
$$

where $C_{1}=10^{-2} \mathrm{ergs}_{\mathrm{cm}^{-3}} \mathrm{~s}^{-1}, y_{c}=3 \mathrm{Mm}$, and $\lambda=3 \mathrm{Mm}$. This localized heating is concentrated in the regions of strong $B_{y}$.

\section{PROMINENCE FORMATION DUE TO EVAPORATION AND THERMAL INSTABILITY}

As the localized heating is functioning, chromospheric plasma is heated and evaporated into the arched coronal loops, increasing the density and the temperature there. About 6 minutes later, the temperature reaches a maximum value of $2.2 \mathrm{MK}$, and then starts to decrease slowly (see Figure 1(c, d)). At about 84 minutes, the temperature of an inverted triangle-shaped region around the apexes of a bundle of magnetic loops decreases drastically (see Figure 1(f)) and a small condensation with the typical chromospheric density $\left(6.3 \times 10^{10}\right.$ $\mathrm{cm}^{-3}$ ) appears near the loop top at a height of $25.4 \mathrm{Mm}$. This is accompanied by two strong inflows moving towards the central condensation segment, with a maximum velocity of $70 \mathrm{~km}$ $\mathrm{s}^{-1}$ from the two sides (see Figure1(e)). The magnetic field lines near the condensation make an angle of $62^{\circ}$ with respect to the $z$-direction. After this sudden birth of the prominence, condensation successively happens on the tops of lower and higher coronal loops, leading to a rapid extension of the prominence in the vertical direction. As the inflows collide near the loop tops, two rebound shock waves are formed (see diamond-shaped wave fronts in Figure 1 $(\mathrm{g}, \mathrm{h})$ ) and propagate from the apex towards the loop feet, during which they sweep across and slow down the evaporated upflows.

In order to investigate the thermal instability during the in-situ formation of the prominence, we quantify the temperature, the time derivative of temperature, the density, the pressure, the thermal instability isochoric criterion $C_{P}$ (Parker 1953) and isobaric criterion $C_{F}$ (Field 1965) at the site $(x=0, y=25.4 \mathrm{Mm})$ of the first condensation (see Figure 2). A value of $20 \mathrm{Mm}$ is adopted as the wavelength of perturbation when calculating the thermal instability criteria (Xia et al. 2011). After $t=82.3$ minutes, when the temperature and pressure start to decrease nonlinearly, both $C_{P}$ and $C_{F}$ dive into the negative region indicating

the functioning of thermal instability. Although the localized heating in the chromosphere is in a wide range in the $x$-direction, plasma condensation appears only on long field loops. The plasma conditions along these field loops satisfy the criterion of thermal instability. At a 
given height, a larger shear rate (quantified by $\mu$ ) leads to a longer field line whose footpoints are closer to the neutral line, which renders the sheared arcade more vulnerable to thermal instability.

\section{OVERALL PROMINENCE STRUCTURE AND FORCE BALANCE}

As the prominence grows fatter and heavier, the field lines penetrating the prominence are gradually bend downwards forming vertically aligned dips. This is shown in Figure 3(a), a 3-dimensional (3D) illustration of the vertical 'sheet-like' prominence and selected field lines at $t=143$ minutes. The right panel of Figure 3 gives the corresponding 2D projected image. At this moment, the prominence has a vertical extension of $12 \mathrm{Mm}$ from its bottom at $15.3 \mathrm{Mm}$ to its top at $27.3 \mathrm{Mm}$. The horizontal width of the prominence is about $2.7 \mathrm{Mm}$ at the middle and very thin at top and bottom edges. In the prominence, the temperature is about $18600 \mathrm{~K}$, the density varies from $7.3 \times 10^{10}$ at the bottom to $4.3 \times 10^{10} \mathrm{~cm}^{-3}$ at the top, and the plasma beta changes from 0.22 to 0.47 as the magnetic field strength changes from $6.8 \mathrm{G}$ at the bottom to $3.7 \mathrm{G}$ at the top. The coronal loops below the prominence are heated to nearly $2 \mathrm{MK}$, which is hotter than the other coronal area $(\sim 1.4 \mathrm{MK})$. The transition region above the neutral line has risen to a height of about $7 \mathrm{Mm}$, which is similar to previous works (Lionello et al. 2001; Mok et al. 2005).

The total mass of the prominence is increasing as the localized chromospheric heating is kept. In order to quantify the prominence mass, we integrate the plasma with density larger than $4.2 \times 10^{10} \mathrm{~cm}^{-3}$ within a square box which surrounds the prominence. Within the first 10 minutes, the prominence mass grows nonlinearly with a mean rate of about 764 $\mathrm{g} \mathrm{cm}^{-1} \mathrm{~min}^{-1}$. Later the prominence mass grows linearly, with a rate of about $519 \mathrm{~g} \mathrm{~cm}^{-1}$

$\mathrm{min}^{-1}$. The temporal evolution of the total mass of the prominence in a unit length in the $z$-direction is quantified in Figure 4 .

The initial magnetic field was force-free, with the current being parallel to the magnetic field. After the formation of the prominence, the current is locally increased in the prominence region. The $z$-component of the current density in the prominence is positive and is significantly stronger than in the surrounding corona, as shown by Figure 5(a). The magnetic field in the plane is pointing from the left to the right, so the Lorentz force in the prominence is pointing upward and is able to balance the gravity of local dense plasma. This is convincingly demonstrated by comparing the gravity, Lorentz force, gas pressure gradient, and their sum, through the prominence structure. We check this first along a central vertical line s1 through the prominence (see Figure 5(a)). The distributions of these forces are displayed in Figure 5(b). Near the prominence-corona transition regions (PCTR) at 15.3 
Mm and 27.6 Mm heights, the gas pressure gradient and Lorentz force fluctuate rapidly, as this transition region is complicated by the local thermodynamics driven by the radiative losses combined with field-aligned heat conduction. In this thin PCTR layer, forces are not in balance exactly, but the average value of the resultant force to remain close to zero. However, in the prominence body, the dominant Lorentz force and gravity nearly balance perfectly, except for small fluctuations of the Lorentz force, which are then compensated by opposite fluctuations of the pressure gradient. The resultant force vanishes throughout the prominence body, as indicated by the solid line, realizing force-balance in the vertical direction. In the horizontal direction, the distributions of the Lorentz force, the pressure gradient, and their resultant force along the slice s2 are plotted in panel (c) of Figure 5 , We find that inside the prominence $(0<x<1.1 \mathrm{Mm})$, the force of the pressure gradient points outwards and almost balanced by the Lorentz force, which is pointing to the center of the prominence. Hence the horizontal balance is realized by a magnetic pinching. The strength of these forces increases from the center to the edge of the prominence, and a small resultant force points outwards. In the thin PCTR region, forces fluctuate again indicating the detailed thermodynamic processes at play there. In the end, the mass of the prominence

would be saturated, after which any newly-formed condensation would fall aside from the corresponding magnetic dips, forming the drainage of cool material.

\section{CONCLUSION}

In this Letter, we simulate the formation of a normal-polarity quiescent prominence in a magnetized coronal arcade by chromospheric heating for the first time in a realistic multi-dimensional magnetic configuration. There should be no fundamental difference in the inverse-polarity configuration, which requires 3D simulations, as far as the radiatively driven condensation is concerned. The magnetic dips supporting the prominence mass against gravity are self-consistently formed in an overall low-beta environment. Therefore, the magnetic dips in our model are a consequence of the prominence formation. Our simulation captures many details relevant to quiescent prominence models, and closely resembles many observational features. The prominence body, elongated along the invariant direction, is situated above the magnetic neutral line and the magnetic field through the prominence makes a finite angle to its axis. Our model naturally produces a vertical 'sheet-like' prominence rather than multiple threads aligned along the magnetic field lines. As long as the chromospheric heating is active, the prominence grows in horizontal and vertical size, while the mass-loaded arcade field loops realize a force balance between Lorentz force and gravity throughout the prominence body. Our simulation radically improves all earlier evaporation-condensation studies, which assume rigid one-dimensional field line shapes along which catastrophic cooling sce- 
narios have been studied in parametric detail, invariably leading to field-aligned prominence threads. Similar parametric studies will be required in our fully multi-dimensional settings, to determine their impact on the macroscopic parameters like prominence width, height and total mass.

This study can guide future observations dedicated to find direct evidence of plasma condensation and mass-supply mechanism in prominences and ultimately uncover these mysteries of prominences. Our model can act as a starting point for future studies of RayleighTaylor instability development in quiescent prominences, extending recent local box studies (Hillier et al. 2011), by allowing for true sheared field configurations. We intend to contrast synthetic views on the obtained prominence structures with modern observations, and will investigate how spatio-temporally varying heating conditions may give rise to multiple condensation sites and filament threads.

C.X. thanks R.L. Jiang, F. Chen, and Y. Guo for discussions. This research was supported by NSFC under grants 11025314, 10878002, and 10933003, and NKBRSF under grant 2011CB811402. Computations used the IBM Blade Center HS22 Cluster at the HPC Center of Nanjing University of China. R.K. acknowledges funding from EC Seventh Framework Programme (FP7/2007-2013) under grant agreement SWIFF (project nr 263340, www.swiff.eu).

\section{REFERENCES}

Amari, T. \& Aly, J. J. 1989, A\&A, 208, 261

An, C.-H., Bao, J. J., Wu, S. T., \& Suess, S. T. 1988, Sol. Phys., 115, 93

Antiochos, S. K., MacNeice, P. J., Spicer, D. S., \& Klimchuk, J. A. 1999, ApJ, 512, 985

Blokland, J. W. S. \& Keppens, R. 2011, A\&A, 532, A93

Bommier, V. \& Leroy, J. L. 1998, in Astronomical Society of the Pacific Conference Series, Vol. 150, IAU Colloq. 167: New Perspectives on Solar Prominences, ed. D. F. Webb, B. Schmieder, \& D. M. Rust, 434

Choe, G. S. \& Lee, L. C. 1992, Sol. Phys., 138, 291

Colgan, J., Abdallah, Jr., J., Sherrill, M. E., Foster, M., Fontes, C. J., \& Feldman, U. 2008, ApJ, 689, 585 
Drake, J. F., Mok, Y., \& van Hoven, G. 1993, ApJ, 413, 416

Field, G. B. 1965, ApJ, 142, 531

Fontenla, J. M., Avrett, E. H., \& Loeser, R. 1991, ApJ, 377, 712

Hildner, E. 1974, Sol. Phys., 35, 123

Hillier, A., Isobe, H., Shibata, K., \& Berger, T. 2011, ApJ, 736, L1

Hood, A. W. \& Anzer, U. 1990, Sol. Phys., 126, 117

Karpen, J. T., Antiochos, S. K., Hohensee, M., Klimchuk, J. A., \& MacNeice, P. J. 2001, ApJ, 553, L85

Keppens, R., Meliani, Z., van Marle, A.J., Delmont, P., Vlasis, A., \& van der Holst, B. 2012, J. Comput. Phys., 231, 718

Kippenhahn, R. \& Schlüter, A. 1957, Zeitschrift fur Astrophysik, 43, 36

Kuperus, M. \& Raadu, M. A. 1974, A\&A, 31, 189

Leroy, J. L., Bommier, V., \& Sahal-Brechot, S. 1983, Sol. Phys., 83, 135

Lionello, R., Linker, J. A., \& Mikić, Z. 2001, ApJ, 546, 542

Low, B. C. 1975, ApJ, 198, 211

-. 1977, ApJ, 212, 234

Mackay, D. H., Karpen, J. T., Ballester, J. L., Schmieder, B., \& Aulanier, G. 2010, Space Sci. Rev., 151, 333

Mandrini, C. H., Démoulin, P., \& Klimchuk, J. A. 2000, ApJ, 530, 999

Mok, Y., Drake, J. F., Schnack, D. D., \& van Hoven, G. 1990, ApJ, 359, 228

Mok, Y., Mikić, Z., Lionello, R., \& Linker, J. A. 2005, ApJ, 621, 1098

-. 2008, ApJ, 679, L161

Parker, E. N. 1953, ApJ, 117, 431

Petrie, G. J. D., Blokland, J. W. S., \& Keppens, R. 2007, ApJ, 665, 830

Sparks, L., van Hoven, G., \& Schnack, D. D. 1990, ApJ, 353, 297 
van der Linden, R. A. M., Goossens, M., \& Hood, A. W. 1992, Sol. Phys., 140, 317 van Hoven, G. \& Mok, Y. 1984, ApJ, 282, 267

Wu, S. T., Bao, J. J., An, C. H., \& Tandberg-Hanssen, E. 1990, Sol. Phys., 125, 277

Xia, C., Chen, P. F., Keppens, R., \& van Marle, A. J. 2011, ApJ, 737, 27

This preprint was prepared with the AAS IATEX macros v5.2. 

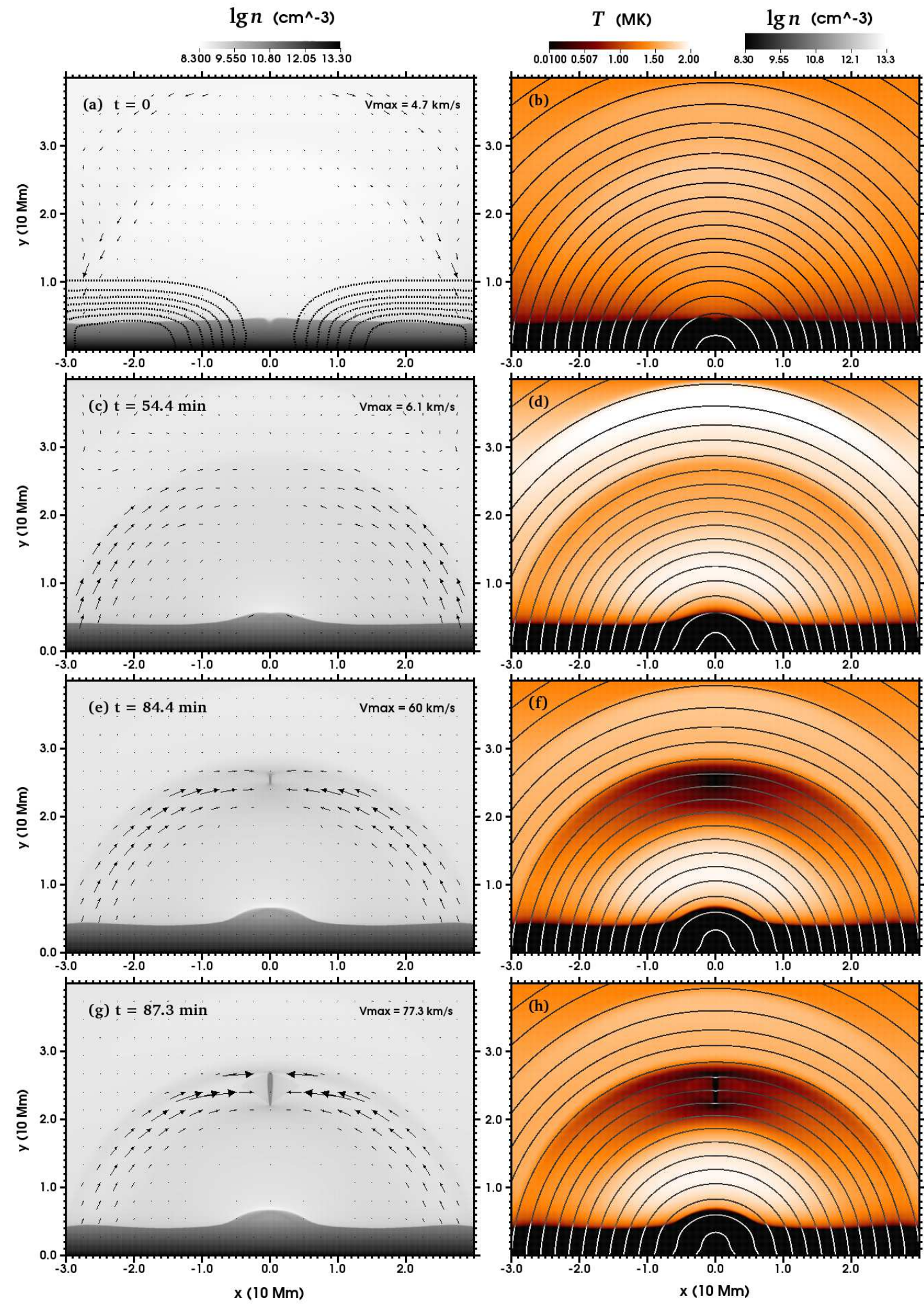

Fig. 1. - Snapshots of the formation process at $t=0$ (first row), 54.4 mins (second row), 84.4 mins(third row), and 87.3 mins (bottom row). In the left column, the density is shown in gray and the projected velocity field is shown by arrows. The right column shows the temperature and the projected field lines are colored by the local density. Contours of the localized heating are plotted in panel (a) in dotted lines. 


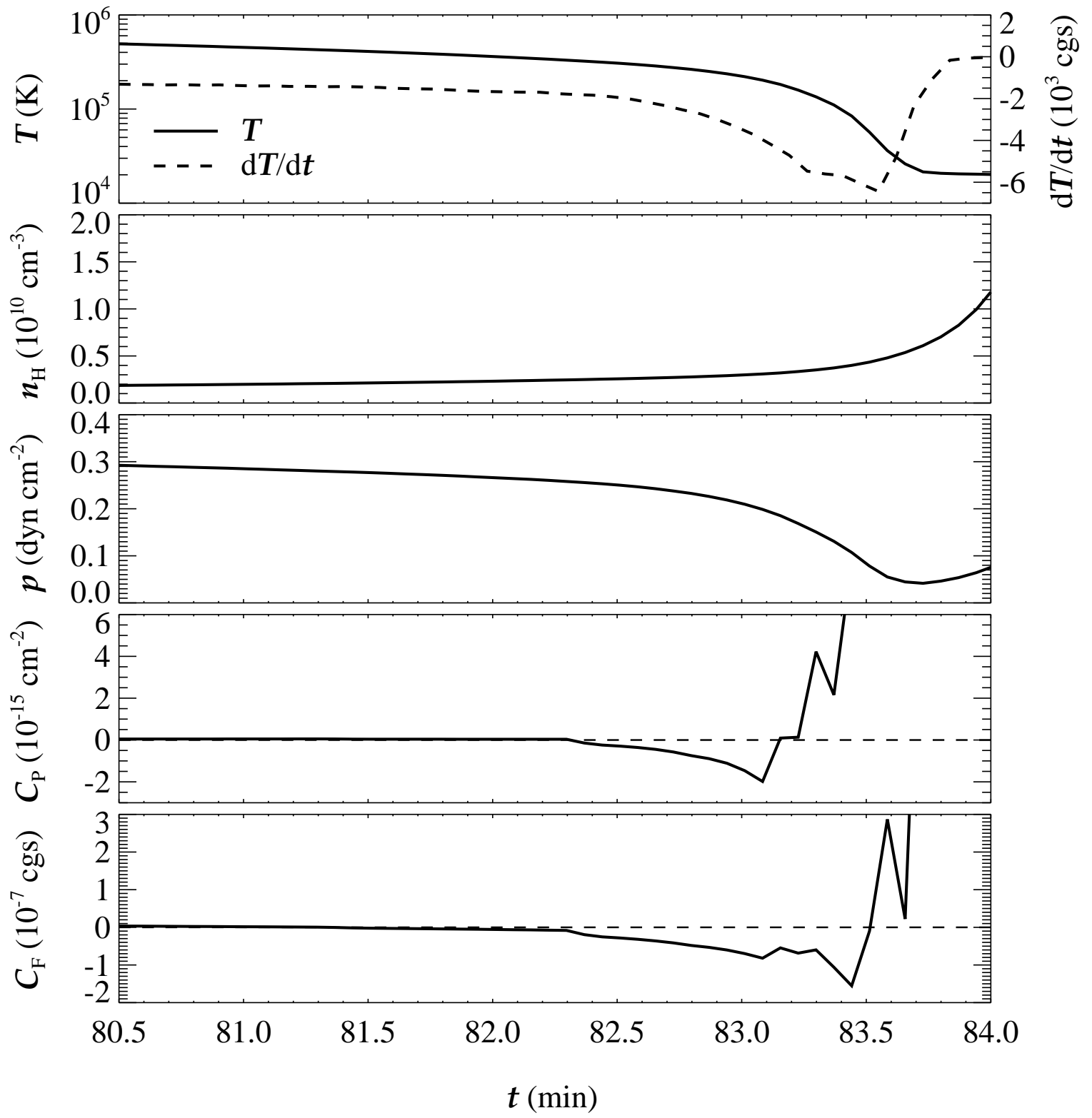

Fig. 2.- Temporal evolutions of the temperature, the density, the pressure, the thermal instability isochoric criterion $C_{P}$, and the isobaric criterion $C_{F}$ at the first condensation site (solid lines), as well as the time derivative of the temperature (dashed line, top panel). The horizontal dashed lines in the last two panels denote the zero value. 

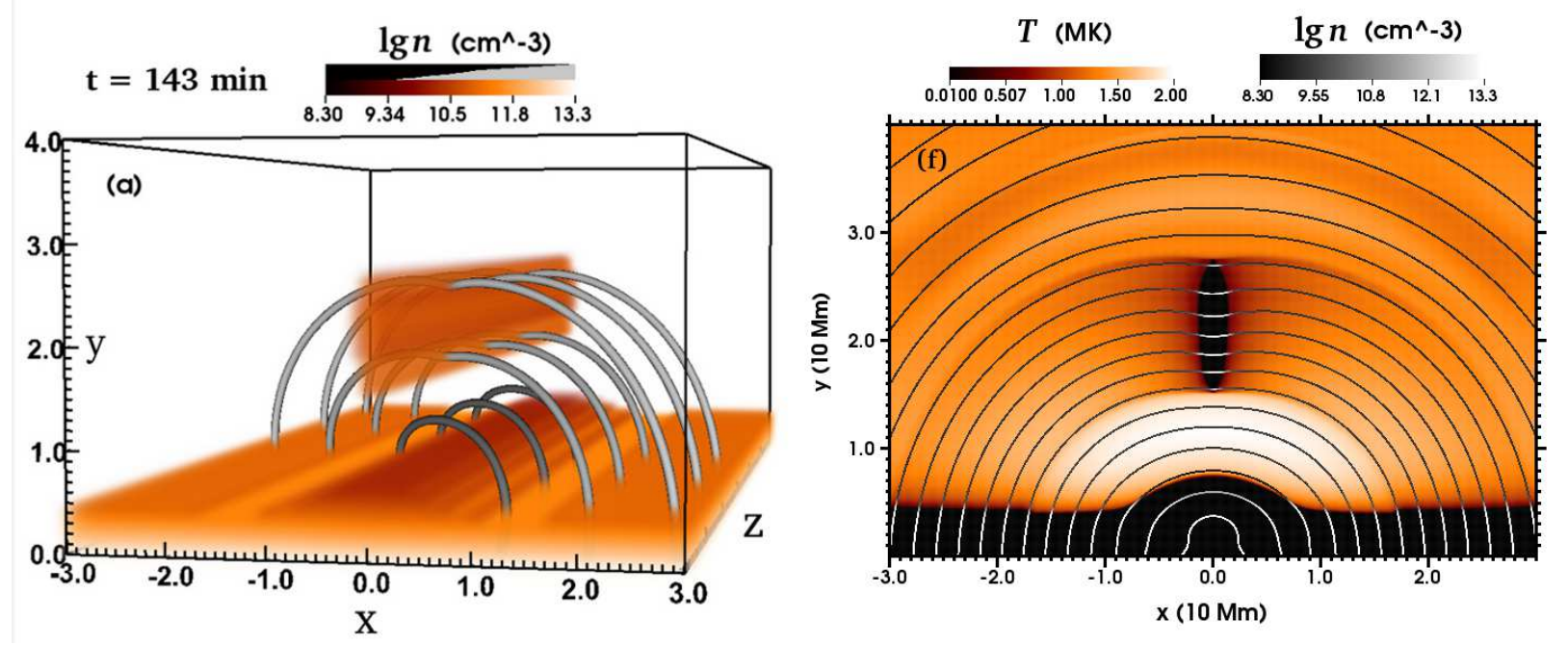

Fig. 3. - A K-S prominence at $t=143$ mins. (a) 3D impression of the prominence shown by the number density and selected magnetic field lines. The bar above the color bar shows the opacity of corresponding colors. (b) the temperature and the projected field lines colored by the local density. 


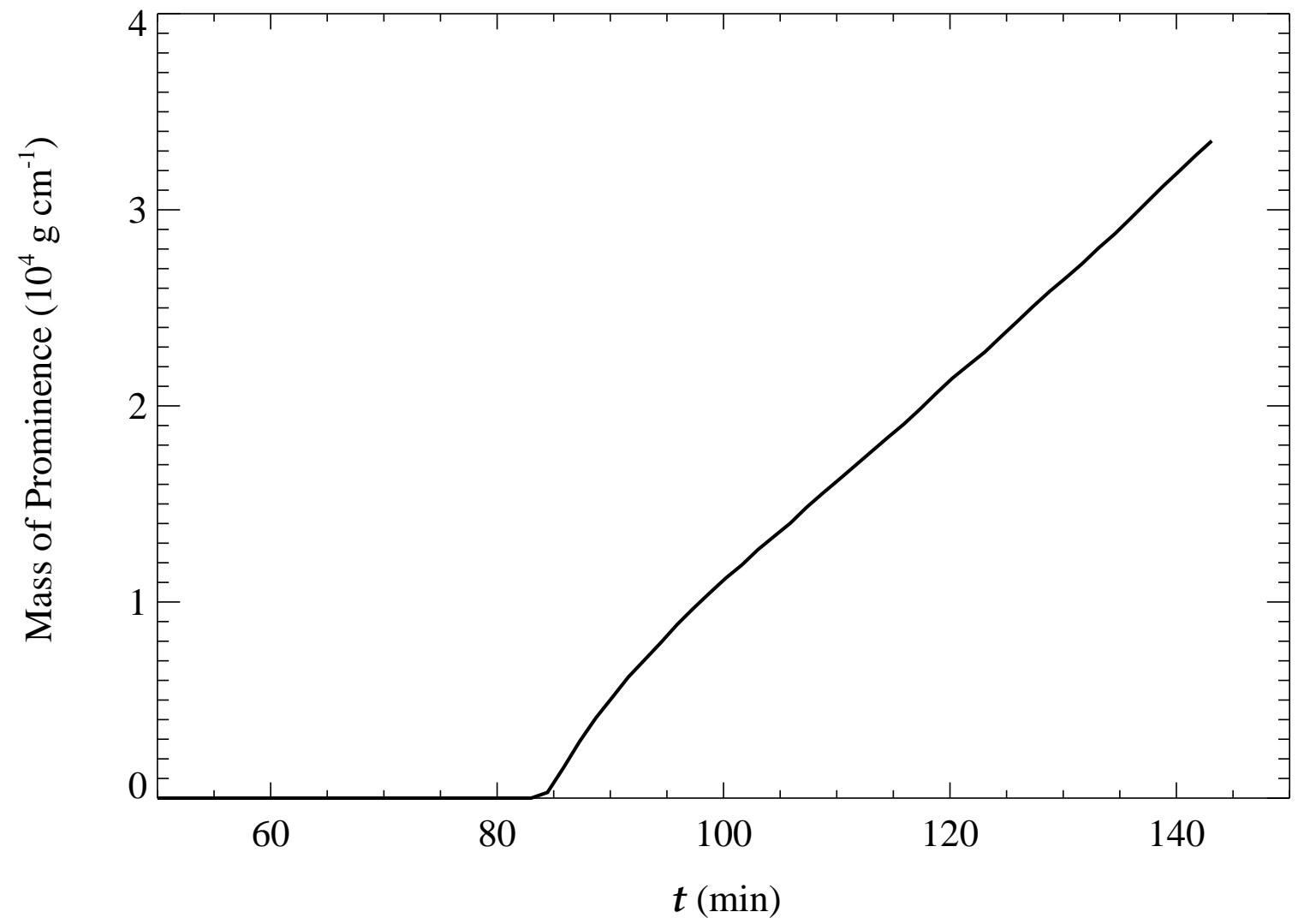

Fig. 4. - Temporal evolution of the prominence mass. 

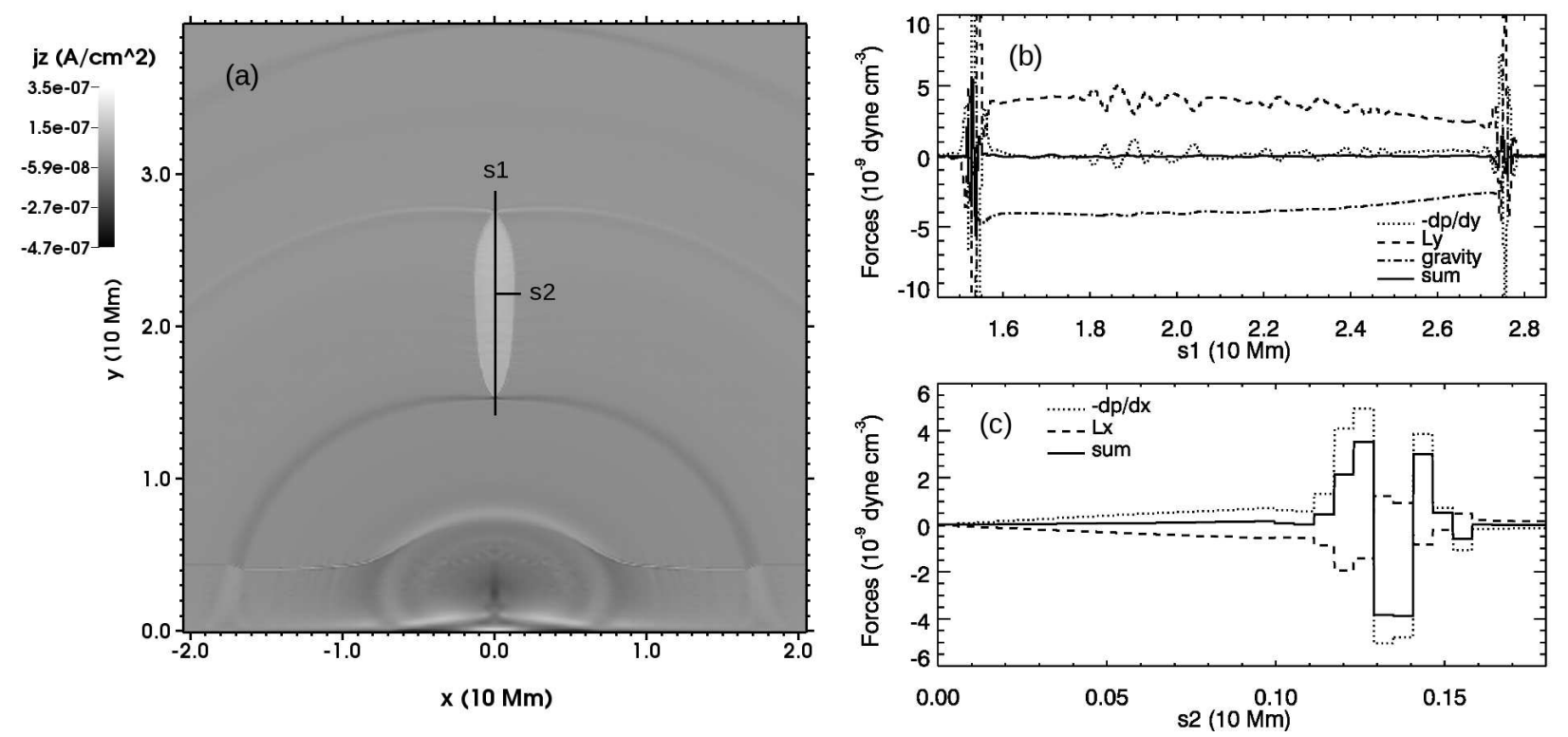

Fig. 5.- Panel (a): Distribution of z-component of the current density $\left(j_{z}\right)$; Panel (b): Distributions of various forces along the vertical slice s1 in panel (a), including the gas pressure gradient (dotted line), Lorentz force (dashed line), gravity (dashed dotted line), and their sum (solid line); Panel (c): Distributions of various forces along the horizontal slice s2 in panel (a), including pressure gradient (dotted line), Lorentz force (dashed line), and their sum (solid line). 